\title{
Modelo Pedagógico ML-SAI: Uma Atividade Experimental no Ensino Médio
}

\author{
Ernane Rosa Martins , Luís Manuel Borges Gouveia² \\ ${ }^{1}$ Instituto Federal de Goiás (IFG) \\ Caixa Postal 72.811-580 - Luziânia - GO - Brasil \\ ${ }^{2}$ Universidade Fernando Pessoa (UFP) \\ Caixa Postal 4249-004 - Porto - Portugal \\ ernane.martinseifg.edu.br, lmbg@ufp.edu.pt
}

\begin{abstract}
This article aims to present the ML-SAI pedagogical model, which was based on the theory of the Inverted Classroom (IC) and aims to guide mlearning activities, reporting their experimentation in a High School activity. Thus, this article defines pedagogical models, the aspects related to $\mathrm{m}$ learning and the IC theory, presents the structure and strategies of the $M L$ SAI. It then describes its application, specifying the pedagogical resources used and the methodological procedures adopted. Finally, it presents the results found, promoting some reflections and considerations about the same.
\end{abstract}

Resumo. Este artigo tem como objetivo apresentar o modelo pedagógico $M L$ SAI, que foi fundamentado na teoria da Sala de Aula Invertida (SAI) e visa orientar atividades de m-learning, relatando sua experimentação em uma atividade do Ensino Médio. Assim, este artigo a princípio define os modelos pedagógicos, os aspectos relacionados à m-learning e a teoria SAI, apresenta a estrutura e estratégias do ML-SAI. Em seguida descreve a sua aplicação, especificando os recursos pedagógicos utilizados e os procedimentos metodológicos adotados. Por fim, apresenta os resultados encontrados, promovendo algumas reflexões e considerações sobre o mesmo.

\section{Introdução}

Com o surgimento de novas tecnologias digitais, tais como os smartphones, e visto que estas estão cada vez mais presentes no processo de ensino, os professores estão percebendo a necessidade da utilização de metodologias de aulas mais atrativas e focadas no m-learning, que é a aprendizagem por meio de dispositivos móveis. O $\mathrm{m}$ learning pode ser definido como sendo um campo emergente, que engloba tecnologias sem fio e computação móvel que permite que a aprendizagem possa ocorrer em qualquer tempo e em qualquer lugar (Wains \& Mahmood, 2008).

A Sala de Aula Invertida (SAI) é uma destas metodologias ativas de aprendizagem que vem ganhando espaço entre os professores, de modo a enfrentar os desafios atuais das mudanças desta nova sociedade. A SAI proporciona mudanças significativas na forma de elaboração e execução das atividades pedagógicas, onde os alunos passam a atuar como protagonistas de seu aprendizado e o professor passa a ser um mediador neste processo (Silva, Lima \& Andriola, 2016). Esse entendimento é 
VIII Congresso Brasileiro de Informática na Educação (CBIE 2019)

Anais do XXV Workshop de Informática na Escola (WIE 2019)

corroborado por Bergmann \& Sams (2018) afirmando que com a SAI "a aula gira em torno dos alunos, não do professor, esse está presente unicamente para prover feedback especializado". Na SAI os alunos apropriam-se do conteúdo de forma antecipada a aula presencial e o tempo em sala de aula são utilizados para promover discussões de modo a sanar as dúvidas.

A SAI é um modelo educacional que se encaixa nessa nova perspectiva de ser utilizada em conjunto com as novas tecnologias e aprendizagem virtual, pois busca encaixar às reais necessidades e interesses dos alunos as mudanças sociais que ocorrem atualmente (Tourón, Santiago \& Díez, 2014). Confirmando o relatório da Unesco (2014), onde argumenta ser ideal que a tecnologia e a educação evoluam lado a lado com as necessidades educacionais. Gómez (2015, p. 29) afirma ainda que "é preciso reinventar a escola para que esta possa desenvolver conhecimentos, habilidades, atitudes, valores e emoções". Sendo assim, este artigo tem como objetivo apresentar o modelo pedagógico ML-SAI, que foi fundamentado na teoria da Sala de Aula Invertida (SAI) e visa orientar atividades de m-learning, relatando sua experimentação em uma atividade do Ensino Médio.

Tendo em vista o objetivo proposto, na seção 2 é definido o que este estudo entende por modelo pedagógico, são discutidos os principais aspectos relacionados à $m$ learning, são apresentados os princípios básicos da Teoria SAI. Na seção 3 é apresentada a estrutura e estratégias do ML-SAI. Na seção 4 é estabelecido o método, as técnicas e procedimentos metodológicos utilizados. Na seção 5, apresenta-se os resultados encontrados e algumas reflexões a respeito da experimentação realizada. Finalizando, a seção 6 apresenta algumas considerações importantes sobre o presente trabalho.

\section{Conceituação Científica}

Nesta seção são contextualizados os modelos pedagógicos, os aspectos relacionados à m-learning e os princípios básicos da SAI.

\subsection{Modelos Pedagógicos}

Modelo é definido como "um sistema figurativo que reproduz a realidade de forma mais abstrata, quase esquemática, e que serve de referência" (Behar, Passerino \& Bernardi, 2007, p.3). Os modelos pedagógicos, podem ser encontrados na literatura com diversas definições, tais como: sinônimos de estratégias de ensino, metodologias de ensino, currículo ou como teorias de aprendizagens, não existindo um consenso a respeito (Behar et al., 2007). Neste estudo, utiliza-se a definição de modelo pedagógico como sendo "um sistema de premissas teóricas que representa, explica e orienta a forma como se aborda o currículo e que se concretiza nas práticas pedagógicas e nas interações professor-aluno-objeto de conhecimento", podendo ser embasado por uma ou várias teorias de aprendizagem, ou suas reinterpretações, discutindo-se este assunto conforme os autores que adotam linhas semelhantes (Behar et al., 2007, p. 4).

Segundo Behar (2009), para a construção de um modelo pedagógico, é necessário definir seus elementos. O modelo pedagógico pode ser baseado em duas ou mais teorias educacionais utilizadas como eixo norteador da aprendizagem e nas experiências pessoais, criando o que é denominado de Modelo Pessoal. Assim, este é 
VIII Congresso Brasileiro de Informática na Educação (CBIE 2019)

Anais do XXV Workshop de Informática na Escola (WIE 2019)

fundamentado em dois elementos: Arquitetura Pedagógica (AP) e Estratégias para a Aplicação da mesma. A AP é a estrutura principal do modelo e as estratégias para a aplicação da AP são as dinâmicas do modelo pedagógico, que dependem do contexto e variáveis que envolvem o processo educativo. Estas ações didáticas direcionam o professor para colocar em pratica a AP em um contexto particular, tornando-a individualizada. A arquitetura pedagógica (AP) é formada basicamente por:

- Aspectos relacionados ao conteúdo, que engloba materiais instrucionais e/ou recursos informáticos utilizados, tais como: objetos de aprendizagem, software e outras ferramentas de aprendizagem;

- Aspectos organizacionais, que é a fundamentação do planejamento ou proposta pedagógica onde estão incluídos os propósitos da aprendizagem, organização do tempo e do espaço e expectativas na relação da atuação dos participantes ou da organização social da classe;

- Aspectos tecnológicos, com a definição da plataforma e suas funcionalidades, ferramentas de comunicação, entre outros;

- Aspectos metodológicos, que envolvem: atividades, interações, procedimentos de avaliação e a organização de todos esses elementos numa sequência didática para a aprendizagem.

No desenvolvimento de um projeto pedagógico eficiente é necessário levar em consideração alguns aspectos importantes da mudança de paradigma, principalmente referente a modalidade de ensino m-learning. A afirmação de Behar $(2009$, p. 21) que diz "O mundo tem como novos pilares os conceitos de tempo e de espaço" é um exemplo destas mudanças. De acordo com Cardoso \& Burnham (2010) seja a pedido dos alunos ou por necessidades técnicas, diversos ajustes devem ser realizados para construir um modelo pedagógico que atenda as expectativas de ensino e aprendizagem.

\subsection{M-learning}

Para Martins et al. (2018) a aprendizagem móvel (m-learning) é um campo de pesquisa emergente e em expansão, devido à crescente mobilidade da sociedade atual. Sendo que, muitos alunos e professores fazem uso de dispositivos móveis de comunicação, para acesso a materiais didáticos, acesso a ambientes de interação entre alunos e professor, acesso à atividades escolares e compartilhamento de arquivos. Os dispositivos móveis de comunicação são uma alternativa para ampliar as possibilidades dos estudantes, proporcionando novas formas para construção e desenvolvimento do conhecimento.

O relatório da Unesco (2014) aponta os benefícios que a utilização da aprendizagem móvel proporciona, entre eles temos: facilita a aprendizagem individualizada, fornece retorno e avaliação imediata, assegura o uso produtivo do tempo em sala de aula, apoia a aprendizagem fora da sala de aula, cria uma ponte entre a aprendizagem formal e a não formal, amplia a educação em diferentes locais, gera facilidade para estudantes com deficiência, permiti a aprendizagem em qualquer lugar e hora, constrói diferentes comunidades de aprendizado e melhora a comunicação entre os alunos e entre os alunos e o professor. 
VIII Congresso Brasileiro de Informática na Educação (CBIE 2019)

Anais do XXV Workshop de Informática na Escola (WIE 2019)

\subsection{Sala de Aula Invertida}

A Sala de Aula Invertida (SAI) é uma metodologia ativa de aprendizagem, onde a prioridade é o aprendizado por parte do aluno, o conceito principal defendido é a realização de estudos por parte dos alunos antes das aulas, por meio de materiais disponibilizados pelo professor, e após a interação com o conteúdo, o aluno poderá retirar suas dúvidas e dificuldades em atividades dentro da sala de aula (Bergmann \& Sams, 2018).

Martins et al. (2019), apresenta como principais vantagens da SAI: pode-se ouvir/ver as aulas inúmeras vezes; facilita o estudo/aprendizagem; torna as atividades em sala mais práticas; mais tempo para praticar em sala de aula; beneficia quem tem dificuldades; possibilidade de uma aprendizagem melhor, uma vez que o aluno não estudará somente na véspera da prova; otimização do tempo de sala de aula e obriga os alunos a estudar. Em contrapartida, segundo os mesmos autores, entre os principais desvantagens da SAI, temos: se não conseguir assistir ao vídeo fica perdido em sala de aula; propício à distração; insegurança, por ser uma proposta diferente; dependência muito grande da autonomia e da responsabilidade do aluno; falta de tempo de alunos que trabalham; problema com impossibilidade de conexão com a Internet; as escolas podem encontrar resistência dos professores em relação a referida metodologia; falta de maturidade dos alunos e não é benéfico para alunos esquecidos.

\section{ML-SAI}

O modelo pedagógico foi construído a partir dos estudos preliminares (pesquisa exploratória com estudos de casos) em conjunto com a revisão bibliográfica realizada. Os estudos de caso exploratórios preliminares, utilizaram os conceitos de Sala de Aula Invertida combinada com alguma tecnologia móvel, como Kahoot, WhatsApp e Facebook. Estes estudos de caráter exploratórios investigaram as possibilidades e potencialidades da utilização da Teoria da Sala de Aula Invertida com o auxílio de Tecnologia Móvel.

Assim, como resultados encontrados nestes estudos preliminares, tiveram destaque: as limitações e dificuldades de ordem financeira e técnica que podem excluir alguns alunos que não dispõem de smartphones, planos de Internet em seus celulares ou mesmo Internet em suas residências, dificultando o uso e principalmente o acesso dos alunos às ferramentas e aos recursos digitais disponíveis; benefícios significativos como o baixo custo, a acessibilidade, a interatividade e a aprendizagem colaborativa; a mediação pelo professor sendo um fator fundamental, propondo temas e estimulando a participação dos alunos, identificando o contexto da sala de aula, dos alunos e da turma, estabelecendo regras e normas para utilização dos dispositivos móveis, deixando claro os objetivos e motivos das atividades propostas, verificando as limitações relevantes e os recursos tecnológicos necessários que serão utilizados, assim como, os papeis do professor e dos alunos neste processo; e por fim, a confirmação de que é fundamental um planejamento bem estruturado por parte do professor.

O modelo pedagógico foi formatado para fornecer algumas sugestões de estratégias a professores e pesquisadores interessados em utiliza-lo, orientando estes no desenvolvimento das atividades de m-learning. Neste estudo a AP foi reestruturada e fundamentada levando em consideração os conceitos da Sala de Aula Invertida, os 
VIII Congresso Brasileiro de Informática na Educação (CBIE 2019)

Anais do XXV Workshop de Informática na Escola (WIE 2019)

aspectos relacionados a utilização dos dispositivos móveis e os estudos exploratórios preliminares realizados, a AP foi estabelecida em seis aspectos, sendo estes: contexto, normatização, papeis, tecnologias, ações e limitações. A AP e as estratégias para a Aplicação da AP definidas para as atividades de m-learning estão apresentadas na Tabela 1.

Tabela 1. Modelo pedagógico proposto: ML-SAI (Martins \& Gouveia, 2019).

\begin{tabular}{|c|c|}
\hline $\begin{array}{c}\text { Arquitetura } \\
\text { Pedagógica (AP) }\end{array}$ & Estratégias para a Aplicação da AP \\
\hline \multirow{2}{*}{ Contexto } & $\begin{array}{l}\text { Definir os objetivos e motivos das atividades e ações proposta, deixando-os claros } \\
\text { para todos os envolvidos; }\end{array}$ \\
\hline & $\begin{array}{l}\text { Identificar os instrumentos, recursos, características das atividades e ações, dos } \\
\text { alunos e do curso; }\end{array}$ \\
\hline \multirow[b]{2}{*}{ Normatização } & Organizar regras e procedimentos para orientar as ações e interações; \\
\hline & $\begin{array}{l}\text { Estabelecer normas para utilização dos dispositivos móveis (quando utilizar, qual a } \\
\text { finalidade, etc.); }\end{array}$ \\
\hline \multirow[t]{2}{*}{ Papeis } & $\begin{array}{l}\text { Compreender o papel do aluno no processo de aprendizagem, suas motivações, } \\
\text { interesses e habilidades; }\end{array}$ \\
\hline & Entender o papel do professor como condutor e facilitador da aprendizagem; \\
\hline \multirow[t]{2}{*}{ Tecnologias } & $\begin{array}{l}\text { Definir os dispositivos móveis, aplicativos e recursos tecnológicos que serão } \\
\text { utilizados, considerando as características físicas, técnicas e funcionais dos } \\
\text { mesmos, tais como: ambiente virtual, Sílabe, Moodle, Facebook, Khan } \\
\text { Academy, YouTube, vídeo-aula, músicas, slides, fotografias, áudios, textos, entre } \\
\text { outros, estabelecendo prioridade para aplicativos livres e gratuitos; }\end{array}$ \\
\hline & Verificar a necessidade e disponibilidade de conexão com a Internet; \\
\hline \multirow{9}{*}{ Ações } & $\begin{array}{l}\text { Especificar se as ações serão individuais, em grupo ou ambas, se estas serão } \\
\text { comuns a todos os alunos ou diferenciadas por aluno ou grupo de alunos; }\end{array}$ \\
\hline & $\begin{array}{l}\text { Definir ferramentas de comunicação e sistemas de apoio para dar suporte aos } \\
\text { alunos em caso de dificuldades; }\end{array}$ \\
\hline & $\begin{array}{l}\text { Definir se as ações serão realizadas em um mesmo local, ao mesmo tempo ou em } \\
\text { locais e momentos distintos; }\end{array}$ \\
\hline & $\begin{array}{l}\text { Estabelecer práticas educacionais favoráveis ao aprendizado (situações problemas, } \\
\text { aplicações práticas, colaborativas, autônomas, críticas, em contextos reais, } \\
\text { pesquisas), levando em consideração os ambientes de aprendizagem (on-line, salas } \\
\text { de aula, laboratórios) de preferência com os dispositivos móveis dos próprios } \\
\text { alunos; }\end{array}$ \\
\hline & $\begin{array}{l}\text { Incentivar a interação entre os alunos e com o professor, por meio do uso de } \\
\text { dispositivos móveis, com foco no desenvolvimento da atividade proposta; }\end{array}$ \\
\hline & $\begin{array}{l}\text { Determinar os mecanismos de avaliação de desempenho e certificação da } \\
\text { aprendizagem, se individuais ou em equipes, de preferência continua, e } \\
\text { disponibiliza-los para os alunos; }\end{array}$ \\
\hline & $\begin{array}{l}\text { Estabelecer momentos de reflexão e análise das atividades realizadas, buscando } \\
\text { colaborar na melhoria continua de novas atividades; }\end{array}$ \\
\hline & $\begin{array}{l}\text { Estruturar os conteúdos que serão disponibilizados em ambiente virtual, para que } \\
\text { os alunos possam acessa-los por meio de um dispositivo móvel, quando e quantas } \\
\text { vezes quiserem, se possível com o acompanhamento das visualizações pelo } \\
\text { professor; }\end{array}$ \\
\hline & $\begin{array}{l}\text { Realizar uma curadoria dos conteúdos já existentes na Internet, por meio de } \\
\text { plataformas como Khan Academy e o YouTube em busca de bons vídeos } \\
\text { educativos, ou caso não sejam encontrados, gravar vídeos ou áudios utilizando as }\end{array}$ \\
\hline
\end{tabular}


VIII Congresso Brasileiro de Informática na Educação (CBIE 2019)

Anais do XXV Workshop de Informática na Escola (WIE 2019)

\begin{tabular}{|l|l|}
\hline & $\begin{array}{l}\text { ferramentas que existem no próprio dispositivo móvel; } \\
\text { Estimular diferentes formas de aprendizado por meio de diferentes fontes de } \\
\text { conteúdo, tais como: vídeos, áudios, imagens, textos, slides, questões, entre outras; } \\
\text { Elaborar um roteiro de atividades do que será feito dentro da sala de aula, de modo } \\
\text { a otimizar o tempo em sala de aula, utilizando projetos, trabalhos ou solução de } \\
\text { problemas, que se conectem com o que foi visto previamente na plataforma; }\end{array}$ \\
\hline $\begin{array}{l}\text { Levantar os principais pré-requisitos das atividades e possíveis distratores do } \\
\text { aprendizado; } \\
\text { Limitações }\end{array}$ & $\begin{array}{l}\text { Verificar se os materiais pedagógicos podem ser utilizados em dispositivos móveis, } \\
\text { considerando tamanho da tela, usabilidade, capacidade de armazenamento e } \\
\text { modelos de dispositivos diferentes; } \\
\text { Verificar a disponibilidade de dispositivos móveis, tomadas para recarregar as } \\
\text { baterias dos celulares, conexão com a Internet, quando necessário, e se os } \\
\text { aplicativos apresentam interface adequada a aprendizagem do conteúdo. }\end{array}$ \\
\hline
\end{tabular}

Observa-se que o modelo pedagógico proposto pode se adaptar e colaborar com o desenvolvimento de diversas atividades de m-learning envolvendo diferentes conteúdos e dispositivos móveis.

\section{Metodologia}

O método utilizado nesta pesquisa foi o estudo de caso, que consiste em uma investigação empírica que estuda um fenômeno em seu contexto real, geralmente, em que os limites deste fenômeno não estão claramente definidos. Este estudo é considerado piloto, pois visa auxiliar o pesquisador a aprimorar os procedimentos a serem adotados em pesquisas posteriores e colaborar no esclarecimento de questões conceituais envolvidas (Yin, 2014). A presente pesquisa caracteriza-se como exploratória. De acordo com Gil (2019), a pesquisa exploratória visa desenvolver, esclarecer ou modificar conceitos e ideias sobre determinado assunto, em geral, tendo em vista estudos posteriores.

Para a coleta de dados, além da técnica de observação das atitudes dos alunos e professores, também optou-se por elaborar e aplicar um questionário junto aos alunos envolvidos. Os dados coletados foram tabulados e analisados com enfoque quantitativo e qualitativo. Desta pesquisa participaram alunos do segundo ano do curso Técnico em Informática para Internet presencial durante o primeiro semestre de 2019, com alunos com idades de 15 e 16 anos, totalizando 30 alunos participantes. Todos os alunos possuíam aparelhos smartphones, sendo estes utilizados na atividade, permitindo maior aproximação com o que os alunos estão habituados a utilizar em seu dia a dia e assim facilitar a aprendizagem. Utilizou-se o Edmodo como ambiente de aprendizagem, por ser o que os alunos já utilizavam nas aulas normais e por estar disponível para acesso por smartphones, por meio de aplicativo.

A observação consistiu nos seguintes aspectos: i) o uso de recursos tecnológicos; ii) o desenvolvimento da atividade individual ou em grupo; iii) discussão dos conteúdos abordados; iv) o entendimento dos papeis, em que o aluno é agente do seu processo de aprendizagem e o professor é mediador do processo de aprendizagem; v) a troca de conhecimentos entre alunos; vi) a responsabilidade dos alunos com sua aprendizagem; vii) a autonomia dos alunos em buscar novos conhecimentos. 
VIII Congresso Brasileiro de Informática na Educação (CBIE 2019)

Anais do XXV Workshop de Informática na Escola (WIE 2019)

\section{Experimentação do ML-SAI}

O primeiro passo da experimentação foi realizar a estruturação da atividade a ser realizada, que teve como objetivo compreender o escalonamento de processos em sistemas em lote e interativos, conforme observado na Tabela 2.

Tabela 2. Estrutura da atividade realizada

\begin{tabular}{|c|c|}
\hline AP & Estratégias para a Aplicação da AP \\
\hline Contexto & $\begin{array}{l}\text { 1.1. Objetivo: O objetivo da atividade é compreender como os algoritmos de } \\
\text { escalonamento escolhem o próximo processo a ser executado; } \\
\text { 1.2. Instrumentos e recursos: Vídeos: } \\
\text { Estado de processos, escalonamento, fila de pronto e fila de } \\
\text { bloqueado: https://www.youtube.com/watch?v=THqcAalbbFU } \\
\text { - Animação do algoritmo FCFS: https://www.youtube.com/watch?v=iiBij98FtHg } \\
\text { - Animação do algoritmo RR: https://www.youtube.com/watch?v=FgvPfjcxg2A } \\
\text { Slides de aula disponíveis por meio do Edmodo; }\end{array}$ \\
\hline Normatização & $\begin{array}{l}\text { 2.1. Os slides sobre algoritmos de escalonamento devem ser previamente estudados; Em } \\
\text { seguida, o livro didático pode ser consultado para tirar dúvidas sobre o escalonamento } \\
\text { de processos. Os vídeos devem ser assistidos com o objetivo de compreender as filas de } \\
\text { pronto e bloqueado e para avaliar o comportamento de dois algoritmos de escalonamento } \\
\text { que constam no material. Os vídeos também devem contribuir para o aprendizado de } \\
\text { termos técnicos em inglês. Por fim, os exercícios sobre escalonamento, disponibilizado } \\
\text { pelo Edmodo, devem ser resolvidos. Por fim, serão discutidos (e não apresentados) os } \\
\text { algoritmos em sala seguido da correção dos exercícios; } \\
\text { 2.2. Os vídeos e os slides devem e podem ser vistos por meio de dispositivos móveis. Os } \\
\text { livros da bibliografia básica estão disponíveis na biblioteca. Os exercícios devem ser } \\
\text { realizados em papel A4 utilizando a tabela disponibilizada no arquivo PDF do próprio } \\
\text { exercício. O grupo dos WhatsApp da turma poderá ser utilizado para discutir o assunto e } \\
\text { tirar dúvidas entre os alunos e com o professor; }\end{array}$ \\
\hline Papeis & $\begin{array}{l}\text { 3.1. Nesta atividade os alunos têm papel ativo no aprendizado e devem estudar o } \\
\text { material indicado e procurar outras formas de compreender o conteúdo como pesquisa } \\
\text { em livros, sites e vídeos; } \\
\text { 3.2. O professor será um mediador para a discussão entre a equipe e a busca pelo } \\
\text { conhecimento. Ao final da atividade, o exercício proposto será utilizado para sanar as } \\
\text { dúvidas sobre o conteúdo, consolidar o conhecimento adquirido e apresentar conceitos } \\
\text { complementares e relacionar o conteúdo com as aulas anteriores e o próximo conteúdo; }\end{array}$ \\
\hline Tecnologias & $\begin{array}{l}\text { 4.1. Podem ser utilizados nesta atividade os celulares, computadores pessoais e } \\
\text { institucionais, papel em formato A4, arquivos de documentos em formato PDF, slides } \\
\text { digitais em formato PDF, vídeos on-line como os disponibilizados pelo YouTube, livros, } \\
\text { softwares e sites para educação à distância como Edmodo; } \\
\text { 4.2. Será necessário a conexão à Internet que pode ser utilizada na própria instituição; }\end{array}$ \\
\hline Ações & $\begin{array}{l}\text { 5.1. Esta ação é individual, contudo a turma deverá utilizar as ferramentas de } \\
\text { comunicação em grupo, como por exemplo o WhatsApp, para discutir o tema da aula de } \\
\text { forma prévia; } \\
\text { 5.2. Em caso de dificuldade antes da aula, as dúvidas podem ser dirimidas por meio do } \\
\text { grupo de WhatsApp com a turma e professor ou pelo e-mail do professor; } \\
\text { 5.3. As ações serão realizadas individualmente a qualquer momento, por escolha dos } \\
\text { alunos, até a data da aula; } \\
\text { 5.4. O exercício que será realizado ao final da aula tem como objetivo sanar as dúvidas } \\
\text { de forma presencial; } \\
\text { 5.5. A avaliação do aprendizado será realizada por meio de um exercício sobre o assunto } \\
\text { na aula seguinte; } \\
\text { 5.6. Roteiro da aula: debate sobre os tipos de fila existentes no escalonamento de } \\
\text { processos; debate sobre os tipos de algoritmos de escalonamento e para quais cenários se } \\
\text { aplicam; resolução do exercício proposto; avaliação do conhecimento adquirido por }\end{array}$ \\
\hline
\end{tabular}


VIII Congresso Brasileiro de Informática na Educação (CBIE 2019)

Anais do XXV Workshop de Informática na Escola (WIE 2019)

\begin{tabular}{|c|l|}
\hline & meio de exercício; \\
\hline \multirow{2}{*}{ Limitações } & $\begin{array}{l}\text { 6.1. Requisitos: computador ou celular, acesso à Internet, impressora e papel A4; } \\
\text { 6.2. O material poderá ser utilizado em computador pessoal ou celular com exceção do } \\
\text { exercício que não se adequa a tela do celular. }\end{array}$ \\
\hline
\end{tabular}

A experimentação foi realizada a partir da atividade proposta que ao final foi aplicado um questionário, que considerou a seguinte escala de avaliação ( 5 - concordo plenamente, 4 - concordo, 3 - não concordo nem discordo, 2 - discordo e 1 - discordo completamente), conforme apresenta a Tabela 3.

Tabela 3. Respostas das principais questões utilizadas

\begin{tabular}{|c|c|c|c|c|c|}
\hline Perguntas & 5 & 4 & 3 & 2 & 1 \\
\hline O uso de diversos recursos tecnológicos contribuiu no aprendizado? & $70 \%$ & $30 \%$ & $0 \%$ & $0 \%$ & $0 \%$ \\
\hline Os Smartphones foram recursos importantes para a aprendizagem? & $20 \%$ & $60 \%$ & $20 \%$ & $0 \%$ & $0 \%$ \\
\hline Os aplicativos do Smartphone utilizados foram fáceis de usar? & $40 \%$ & $60 \%$ & $0 \%$ & $0 \%$ & $0 \%$ \\
\hline $\begin{array}{l}\text { O acesso aos conteúdos fora da sala de aula pelo Smartphone, foi } \\
\text { satisfatório? }\end{array}$ & $30 \%$ & $60 \%$ & $10 \%$ & $0 \%$ & $0 \%$ \\
\hline A proposta de uso do Smartphone, foi importante para a disciplina? & $10 \%$ & $70 \%$ & $20 \%$ & $0 \%$ & $0 \%$ \\
\hline $\begin{array}{l}\text { A estratégia de disponibilizar materiais diversos referentes ao conteúdo } \\
\text { antes da aula contribuiu para o bom andamento da disciplina e para o } \\
\text { aprendizado? }\end{array}$ & $30 \%$ & $70 \%$ & $0 \%$ & $0 \%$ & $0 \%$ \\
\hline $\begin{array}{l}\text { A atividade realizada em sala com o auxílio do professor, colaborou } \\
\text { para a aprendizagem do conteúdo? }\end{array}$ & $80 \%$ & $20 \%$ & $0 \%$ & $0 \%$ & $0 \%$ \\
\hline Sua participação foi séria e comprometida? & $50 \%$ & $50 \%$ & $0 \%$ & $0 \%$ & $0 \%$ \\
\hline A ajuda dos colegas de turma foi importante para a sua aprendizagem? & $30 \%$ & $60 \%$ & $10 \%$ & $0 \%$ & $0 \%$ \\
\hline A atuação do professor foi importante para sua aprendizagem? & $60 \%$ & $40 \%$ & $0 \%$ & $0 \%$ & $0 \%$ \\
\hline $\begin{array}{l}\text { A metodologia utilizada permitiu aos alunos com dificuldade melhor } \\
\text { aprendizado? }\end{array}$ & $20 \%$ & $70 \%$ & $10 \%$ & $0 \%$ & $0 \%$ \\
\hline A metodologia utilizada favoreceu o aprendizado? & $20 \%$ & $70 \%$ & $10 \%$ & $0 \%$ & $0 \%$ \\
\hline A metodologia proporcionou maior autonomia e interesse dos alunos? & $30 \%$ & $70 \%$ & $0 \%$ & $0 \%$ & $0 \%$ \\
\hline A metodologia utilizada estimulou a participação dos alunos? & $40 \%$ & $60 \%$ & $0 \%$ & $0 \%$ & $0 \%$ \\
\hline Foram utilizados recursos tecnológicos adequados? & $30 \%$ & $40 \%$ & $30 \%$ & $0 \%$ & $0 \%$ \\
\hline Os conteúdos estavam adequados à plataforma de aprendizagem? & $10 \%$ & $70 \%$ & $20 \%$ & $0 \%$ & $0 \%$ \\
\hline A atividade proposta alcançou o objetivo determinado? & $30 \%$ & $50 \%$ & $20 \%$ & $0 \%$ & $0 \%$ \\
\hline
\end{tabular}

O modelo facilitou as interações entre o professor e os alunos, aumentando a participação e o número de perguntas sobre os temas abordados. Os alunos aprovaram a utilização de vídeos on-line para apresentar o conteúdo, permitindo aos alunos controlar quando vê-los, revê-los e se necessário controlar a velocidade de exibição. $\mathrm{O}$ aumento do tempo livre em sala de aula, agradou tanto estudantes quanto o professor, permitindo aprofundar o tema da aula e desenvolver outros assuntos também importantes. $\mathrm{O}$ professor percebeu que pode ter maior percepção das dificuldades dos alunos e assim utilizar este tempo a mais de aula presencial para ajudá-los.

Entre os principais desafios revelados na implementação do modelo incluem: necessidade de mais tempo para preparação das aulas; dificuldade na aceitação do modelo tanto por parte do professor quanto dos alunos; a necessidade dos smartphones e da disponibilidade de Internet; e a necessidade de gerar motivação nos alunos para se prepararem antes da aula. Quando perguntado sobre quais os recursos os alunos 
VIII Congresso Brasileiro de Informática na Educação (CBIE 2019)

Anais do XXV Workshop de Informática na Escola (WIE 2019)

costumam fazer uso com seus Smartphones, responderam: Câmera, calculadora, navegador, lanterna, Clima, iMovie, Aplicativos, Comunicação, jogos, Internet, redes sociais e fazer pesquisas. A Tabela 4 apresenta como os alunos já utilizaram o Smartphone nos estudos anteriormente a esta atividade.

Tabela 4. Utilização de Smartphones em estudos

Utilização do Smartphone para Estudo de Algum Tema Educacional

Sim, vídeo aulas e alguns textos buscados no navegador! Ajudaram bastante!

Sim. Em várias disciplinas recorri a vídeo aulas e alguns textos extras, o que contribui para o aprendizado.

Sim. Para assistir vídeo aulas e ler textos em PDF.

Sim. Já utilizei meu Smartphone para assistir a vídeos no YouTube que pudessem me auxiliar nos estudos.

Sim. Acesso os slides, vídeos aulas, e outros recursos.

Todas as matérias possíveis. SO, BD, Programação, Português, Matemática, História, Inglês, Geografia,

Sociologia, Filosofia, Química, Física, Educação Física, Arte... Acho que fica mais fácil de achar o

conteúdo desejado com vários tipos de explicações diferentes.

Sim, quase todas e foi bastante produtivo

Sim. Edmodo, brainly, sites do google... Ajudou muito e facilitou os estudos.

Sim, uso ele bastante para atividades escolares.

Os alunos investigados afirmaram que estão familiarizados com a utilização do smartphone na educação, em diversas disciplinas e de várias formas, tais como: com a utilização de vídeo aulas, pesquisas textuais, vídeos do YouTube, slides das disciplinas, leituras de PDF, ambiente virtual Edmodo e sites de busca. A Tabela 5 apresenta os comentários relacionados à metodologia pedagógica utilizada pelo professor.

Tabela 5. Comentários dos alunos

\begin{tabular}{|l|}
\hline \multicolumn{1}{|c|}{ Comentários Relacionados à Metodologia Pedagógica Utilizada } \\
\hline A nova metodologia nos auxilia a sermos mais autônomos e capacitados. \\
\hline Essa metodologia foi completamente didática e nos proporcionou melhor fixação dos conteúdos. \\
\hline Esta metodologia é de estrema utilidade para o bom rendimento do aluno. \\
\hline Gosto deste tipo de aula, pois facilita o aprendizado instigando o aluno a participar mais da aula! \\
\hline Essa metodologia é satisfatória, pois te estimula a estudar! \\
\hline O conteúdo poderia ser mais simples, e menos confuso. \\
\hline O recurso utilizado, fez com que tivéssemos mais interesse pela matéria, e interagíssemos com o professor. \\
\hline
\end{tabular}

Todos os alunos consideraram que os dispositivos móveis podem colaborar para fins educativos. Também foi possível indicar, uma boa aceitação do modelo pedagógico adotado, destacando algumas características interessantes, tais como: autonomia dos alunos, auxilio na fixação dos conteúdos, melhorou o rendimento dos alunos, instigou os alunos a maior participação em sala de aula, estimulou o estudo, despertou o interesse pela matéria e aumentou a interação entre os alunos e o professor. O maior tempo para o trabalho prático em sala de aula foi destacado como um ponto extremamente positivo da metodologia adotada tanto por alunos como pelo professor. Assim o trabalho realizado em sala de aula pode envolver uma situação-problema, com o objetivo de transformar simples conceitos em uma tarefa prática que necessitava de encontrar alguma forma de solução, percebeu-se que os alunos desenvolveram muito mais com a realização deste tipo de tarefa. As dúvidas foram dirimidas em um grupo do WhatsApp favorecendo a 
VIII Congresso Brasileiro de Informática na Educação (CBIE 2019)

Anais do XXV Workshop de Informática na Escola (WIE 2019)

interação entre os alunos. A atividade gerou uma certa competição entre eles na busca por novas soluções práticas para o problema proposto, percebendo o aumento do comprometimento dos estudantes com este tipo de atividade. $\mathrm{O}$ apoio constante e presença do professor na atividade, também foi muito elogiada por todos, visto que o professor ganhou muito mais tempo para ajudar nas indagações dos alunos, principalmente socorrendo os que mais precisavam de reforço.

\section{Considerações Finais}

Este trabalho teve como objetivo apresentar o modelo pedagógico ML-SAI, que foi fundamentado na teoria SAI e visa orientar atividades de m-learning, relatando sua experimentação em uma atividade do Ensino Médio. Analisando a implementação do modelo, foi possível observar que as orientações do ML-SAI estão adequadas ao seu objetivo, que é contribuir com orientações para o planejamento e a realização de ações de m-learning por professores e pesquisadores de diferentes áreas. Verificou-se que embora a utilização do modelo exija maior planejamento, os benefícios alcançados são muito interessantes tanto para os alunos quanto para o professor, indicando uma receptividade favorável ao modelo. Posteriormente, pretende-se realizar novas experimentações em outras turmas e níveis de escolaridade, de modo a validar e consolidar o modelo pedagógico desenvolvido. Por fim, pretende-se divulga-lo e disponibiliza-lo em forma digital, para possibilitar a sua ampla utilização.

\section{Referências}

Behar, P. A. (2009). Modelos pedagógicos em educação a distância. Porto Alegre: Artmed, 311 p.

Behar, P. A., Passerino, L., and Bernardi, M. (2007). Modelos Pedagógicos para Educação a Distância: pressupostos teóricos para a construção de objetos de aprendizagem. Revista Novas Tecnologias na Educação, Porto Alegre, v.5, n. 2, p. 1-12.

Bergmann, J., and Sams, A. (2018). Sala de aula invertida: uma metodologia ativa de aprendizagem. Rio de Janeiro: LTC, 116 p.

Cardoso, A. L. M. S., and Burnham, T. F. (2010). Efetividade de um Modelo Pedagógico para um Ambiente Virtual de Aprendizagem. Simpósio Brasileiro de Informática na Educação, p. 1-10.

Gil, A. C. (2019). Métodos e técnicas de pesquisa social. São Paulo: Editora Atlas, 7 eds. 248 p.

Gómez, A. I. P. (2015). Educação na era digital - a escola educativa. Porto Alegre: Editora Penso; $1^{\mathrm{a}}$ ed., $192 \mathrm{p}$.

Martins, E. R., Geraldes, W. B., Afonseca, U. R., and Gouveia, L. M. B. (2018). Tecnologias Móveis em Contexto Educativo: uma Revisão Sistemática da Literatura. RENOTE: Revista Novas Tecnologias na Educação, v. 16, n. 1, p. 1-10.

Martins, E. R., Gouveia, L. M. B., Afonseca, U. R., and Geraldes, W. B. (2019). Experiências em Ensino de Ciências, v. 14, n. 1, p. 522 - 530.

Martins, E. R.; Gouveia, L. M. B. (2019). M-Learning e Sala de Aula Invertida: Construção de um Modelo Pedagógico (ML-SAI). In: Solange Aparecida de Souza Monteiro. (Org.). Inquietações e proposituras na formação docente. 1ed. Ponta Grossa, PR: Atena Editora, v. 1, p. 184-192.

Silva, F. C. M., Lima, A. S., and Andriola, W. B. (2016). Avaliação do suporte de TDIC na formação do pedagogo: Um estudo em Universidade Brasileira. REICE. Revista Iberoamericana sobre Calidad, Eficacia y Cambio en Educación, v. 14, n. 3, p. 77-93.

Tourón, J., Santiago, R., and Díez, A. (2014). The Flipped Classroom: Cómo convertir la escuela en un espacio de aprendizaje. Barcelona: Grupo Océano, Editora Digital Text; 1 Ed., 178 p.

Unesco. (2014). O Futuro da aprendizagem móvel: implicações para planejadores e gestores de políticas. Brasília: UNESCO.

Wains, S. I., and Mahmood, W. (2008). Integrating m-learning with e-learning. 9th ACM SIGITE Conference on Information Technology Education, Cincinnati, USA, p. 31-38.

Yin, R. K. (2014). Estudo de Caso: Planejamento e métodos. Porto Alegre: Bookman, $5^{\mathrm{a}}$ ed., 320 p. 